\title{
ZONIFICACIÓN GEOTÉCNICA DE LOS SUELOS DE LA CIUDAD DE VALLEDUPAR MEDIANTE UTILIZACIÓN DE UN SIG.
}

\author{
Olga Vanessa Palacio Pacheco 1, Gustavo Cadena Carrillo ${ }^{2}$, Eberto Rafael Ortega Sinning ${ }^{3}$, Angélica \\ Patricia Vanegas Padilla ${ }^{4}$
}

\begin{abstract}
${ }^{1}$ Ingeniera Ambiental y Sanitaria Valledupar, Colombia. Contacto: ovpalacio@unicesar.edu.co
2Ingeniero Ambiental y Sanitario Valledupar, Colombia. Contacto: gcadenac007@gmail.com.

3 ingeniero Civil, Magister en Geotecnia. Esp. En Vías y transporte. Docente de la Universidad Popular del Cesar. Valledupar, Colombia. Contacto: ebertoortega@unicesar.edu.co.

4 Ingeniera Ambiental y Sanitaria, Esp. Gestión Ambiental. Magíster en Desarrollo Sostenible y Medio Ambiente. Contacto: angelicavanegasp@unicesar.edu.co. https://orcid.org/0000-0001-5087-6172
\end{abstract}

\section{RESUMEN}

Se realizó la zonificación geotécnica de los suelos en Valledupar (Cesar) mediante la recolección de datos en diversos laboratorios de suelos y obtención de muestras. Se creó la base de datos para la investigación. Los tipos de suelos encontrados fueron SC, SM y GW identificados en todas las comunas de la ciudad. El nivel freático se encontró a partir 1,2 metros de profundidad. El documento contiene la información necesaria para los estudios geotécnicos de la zona donde se concluye que la ciudad puede soportar cimientos para edificación de más de 10 niveles; en zonas con altos porcentajes de grava, estas áreas serán las de mayor incidencia a la hora de aumentar la cobertura de redes de acueducto y alcantarillado. Es el primer estudio investigativo geotécnico a través de modelos de información geográfica en Valledupar; esto indica la generación de un recurso técnico y bibliográfico de rápida consulta.

Palabras clave: Cartografía, geotecnia, tipos de suelos, SIG.

Recibido: 01 de abril de 2021. Aceptado: 03 de Mayo de 2021

Received: April 01, 2021. Accepted: May 03, 2021

DOI: http://dx.doi.org/10.33571/rpolitec.v17n33a9

\section{GEOTECHNICAL ZONING OF THE SOILS OF THE CITY OF VALLEDUPAR USING A GIS}

\begin{abstract}
The geotechnical zoning of the soils was carried out in Valledupar (Cesar) by collecting data in various soil laboratories and obtaining samples. The research database was created. The types of soils found were SC, SM and GW identified in all the communes of the city. The water table was found from a depth of 1.2 meters. The document contains the necessary information for geotechnical studies of the area where it is concluded that the city can support building foundations of more than 10 levels, in areas with high percentages of gravel, these areas will be the ones with the highest incidence when increasing coverage of aqueduct and sewerage networks. It is the first geotechnical research study through geographic information models in Valledupar; This indicates the generation of a technical and bibliographic resource for quick consultation.
\end{abstract}

Keywords: mapping; geotechnics; types of soils, GIS.

Cómo citar este artículo: O. V. Palacio-Pacheco, G. Cadena-Carrillo, E. R. Ortega-Sinning, A. P. VanegasPadilla. (2021). Zonificación geotécnica de los suelos de la ciudad de Valledupar mediante utilización de un SIG," Revista Politécnica, vol. 17, no. 33, pp. 109-119, 2021. DOI: http://dx.doi.org/10.33571/rpolitec.v17n33a9 


\section{INTRODUCCIÓN}

Según el En el censo de 2005, la población en la ciudad de Valledupar era de 354.449 habitantes, y las proyecciones para el año 2019 rondan los 493.342 habitantes [1], lo que muestra que hay un crecimiento significativo en la población, y ha conllevado al aumento apresurado en la construcción de viviendas, al aumento en la cobertura de las vías, redes de acueducto y alcantarillado a lo largo y ancho de toda la ciudad. Por lo que se hace necesario conocer cómo están constituidos los suelos, ya que, para la realización de este tipo de obras e infraestructuras según las normas colombianas aplicables a cada situación, es necesaria la realización de estudios previos en las áreas a tratar, por lo que es preciso contar con una herramienta que nos ayude a constatar la distribución de los suelos en la ciudad, facilitándonos así la toma de decisiones [4].

Por lo cual se consideró necesaria la realización de una zonificación geotécnica de la ciudad de Valledupar. En 2017, se realizó como proyecto de grado una caracterización geotécnica del subsuelo en la zona norte de la cabecera municipal de Valledupar, tomando dos muestras en 30 apiques en la zona en cuestión, en dicho proyecto se evidenciaron algunas de sus características [2]. Con esta información se comenzó a realizar una base de datos para la ciudad de Valledupar, debido a que históricamente no se ha contado con estudios de zonificación de suelos que permitan el conocimiento de su estado [12].

Un sistema de información geográfica (SIG) es un sistema empleado para describir y categorizar la Tierra y otras geografías con el objetivo de mostrar y analizar la información a la que se hace referencia espacialmente [3] y mediante su utilización se llegó a un modelo conceptual avanzado que dio a conocer la problemática geotécnica o geológica del suelo, con el cual se puede realizar estudios preliminares para las obras tales como adecuaciones, amplificaciones y/o reposiciones de acueducto y alcantarillado, entre otras obras ingenieriles [9] .

\section{MATERIALES Y MÉTODO}

Área de estudio: La investigación busca abarcar la ciudad de Valledupar sin contemplar veredas, corregimientos y/o área metropolitana.

Etapa 1: Revisión de la información técnica e instrumentos de recolección de la información.

Creación de la base de datos: para esto se recolectó información secundaria, en los diferentes laboratorios que efectuaron estudios de las características geomecánicas de los suelos en la ciudad de Valledupar.

Identificación de áreas de muestreo: se ubicaron los puntos anteriormente recolectados en un mapa en Google Earth Pro, lo que facilitó la ubicación de las áreas donde se realizaron los muestreos.

Identificación de los puntos de muestreo: luego de identificadas las áreas, estas fueron visitados y según sus características se identificaron los puntos de fácil acceso para la toma y recolección de muestras.

Pruebas de campo: se realizó el reconocimiento de las áreas y la recolección de las muestras. Se realizaron apiques a 1-2 m, se tomaron duplicados de las muestras y se realizaron los siguientes ensayos:

Análisis granulométricos

Clasificación de suelos en los AASTHO y SUCs

Calculo de la capacidad de carga admisible

Ángulo de fricción interna

Determinación de límite líquido

Determinación de límite plástico

Determinación de resistencia al corte [5].

Se incluyó información como el nivel freático encontrado dentro del alcance de la profundidad de los apiques. 


\section{Etapa 2: Procesamiento de datos}

En esta fase se completó la base de datos con los resultados de laboratorio y demás información recolectada, luego se procesó en un sistema de información geográfica con el método de interpolación IDW. Con lo cual se logró la elaboración de la representación geotécnica de los suelos [7].

\section{Etapa 3: Análisis y discusión de los resultados}

Se analizaron las áreas geotécnicas en función del uso del suelo basados en los requerimientos necesarios para los estudios preliminares que pide el Reglamento Técnico del Sector de Agua Potable y Saneamiento Básico, mostrando las características más relevantes de las comunas de la ciudad, incluyendo la identificación de los tipos de suelos, la profundidad del nivel freático, entre otras [6].

\section{RESULTADOS}

Tipificación de suelos de la ciudad de Valledupar mediante el sistema unificado de clasificación de suelos (SUCs).

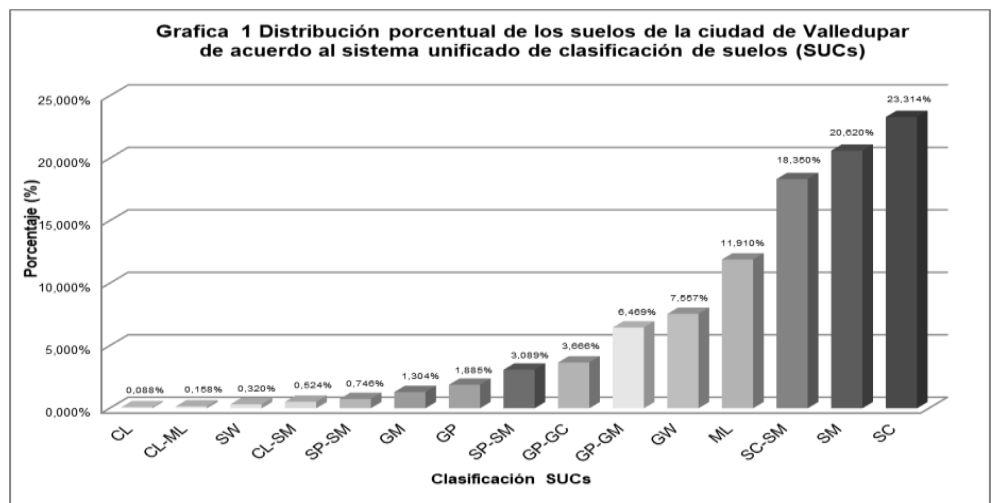

Figura 1. Distribución porcentual de los suelos de la ciudad de Valledupar de acuerdo al sistema unificado de clasificación de suelos (SUCs).

El área de estudio es de 5089,593 $\mathrm{Ha}$, de los cuales predomina un tipo de suelos SC (arenas mal graduadas, arenas con gravas, con poco o nada de fino /arenas limosas, mezcla de arena y limo), según la clasificación del Sistema Unificado de clasificación de Suelos (SUCs) [4] con un 23,314\% del área de estudio que en relación con el área total arroja un equivalente de 1186,5752 Ha.

La ciudad de Valledupar está distribuida en 6 comunas las cuales se tienen en cuenta en el desarrollo del proyecto, por lo cual se realizó la interpretación de los resultados de acuerdo a esto: 


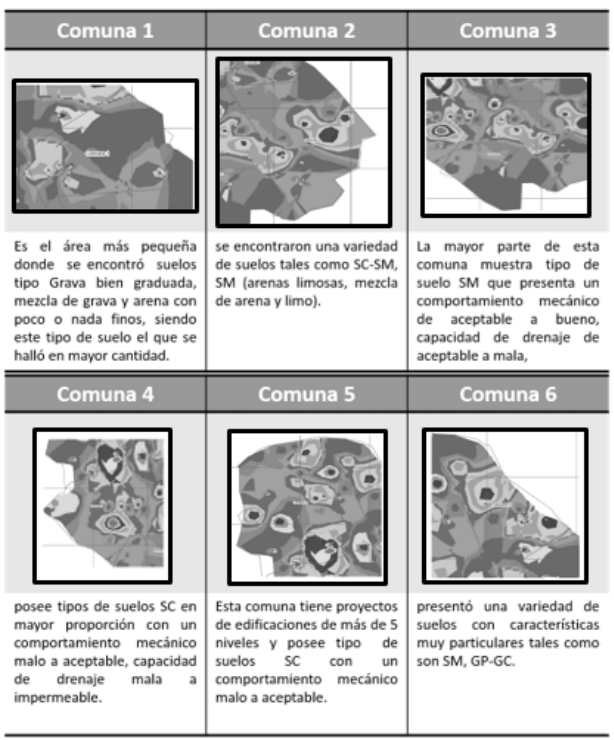

Tabla 1 Distribución por comunas de los suelos de la ciudad de Valledupar de acuerdo al sistema unificado de clasificación de suelos (SUCs).

\section{Tipificación de los suelos de la ciudad de Valledupar mediante la American Association of State} Highway Officials (AASTHO).

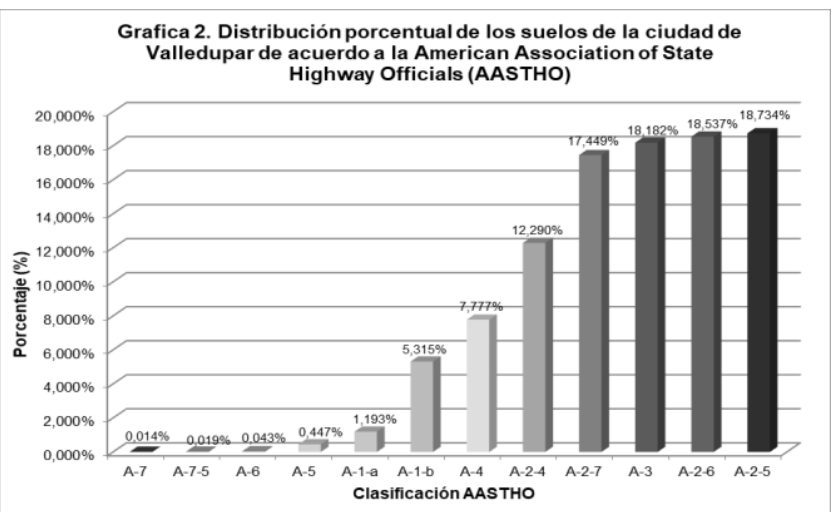

Figura 2. Distribución porcentual de los suelos de la ciudad de Valledupar de acuerdo a la American Association of State Highway Officials (AASTHO).

En la ciudad de Valledupar se puede encontrar suelos del tipo A-7 un área de 0,7125 Ha en relación con un área de estudio total que es de 5089,593 $\mathrm{Ha}$, siendo este tipo de suelo los que menos se encuentran en la zona, de igual forma tipo de suelo A-2-5 es el que se encuentra en mayor parte de esta área de estudio que es de 953,474 Ha, en la siguiente tabla se encuentran las áreas para cada tipo de suelo encontrados en la ciudad según esta clasificación. 


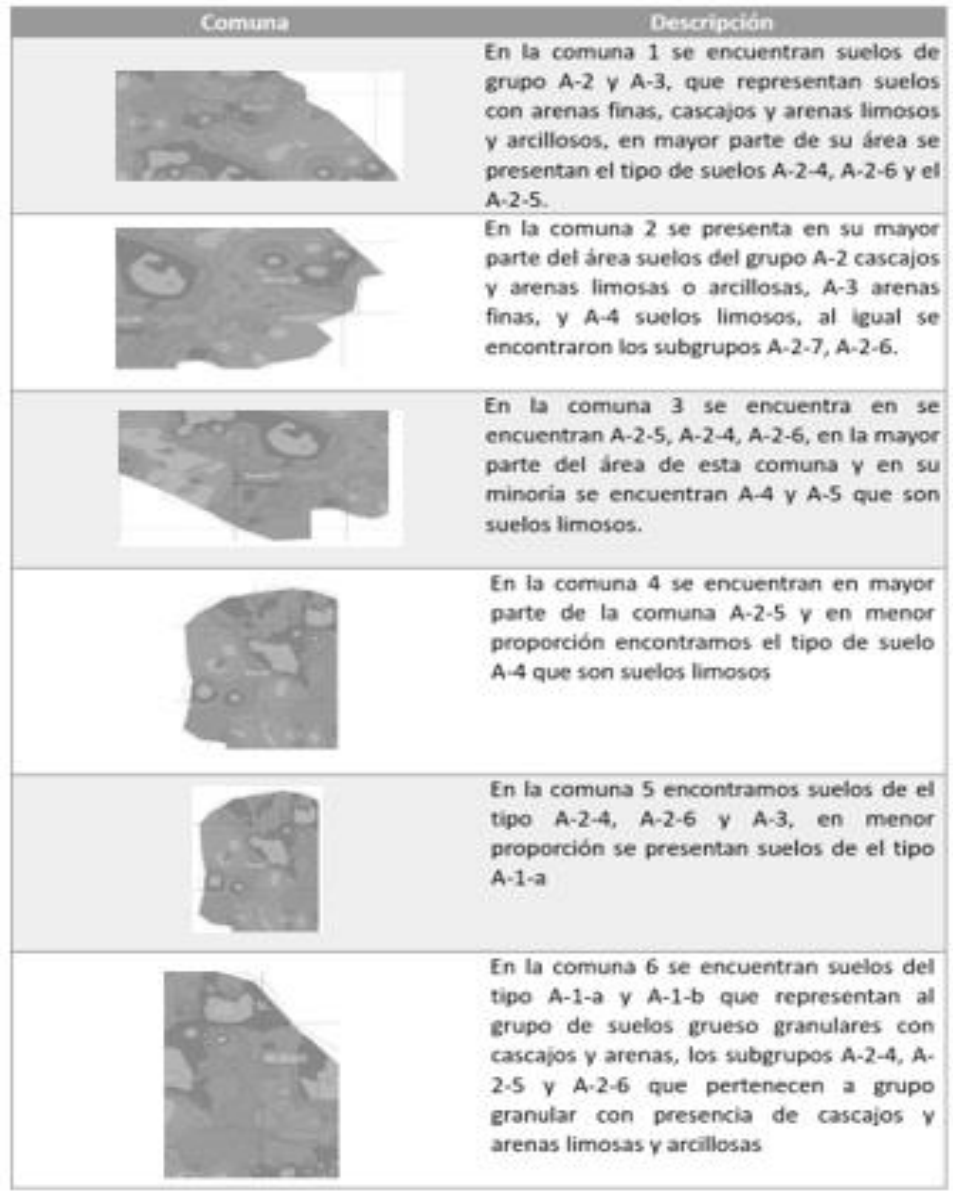

Tabla 2 Distribución por comunas de los suelos de la ciudad de Valledupar de acuerdo a la American Association of State Highway Officials (AASTHO).

\section{Profundidad del nivel freático en la ciudad de Valledupar}

Valledupar es una de las ciudades que cuentan con grandes cantidades de aguas subterráneas, por lo que tiene suelos con capacidades de drenajes buenas y permeabilidad en el subsuelo, llevando el agua de lluvia a zonas de recarga, por lo que se puede encontrar el nivel freático a profundidades de 1,2 $\mathrm{m}$ en barrios como Garupal (comuna 5). 


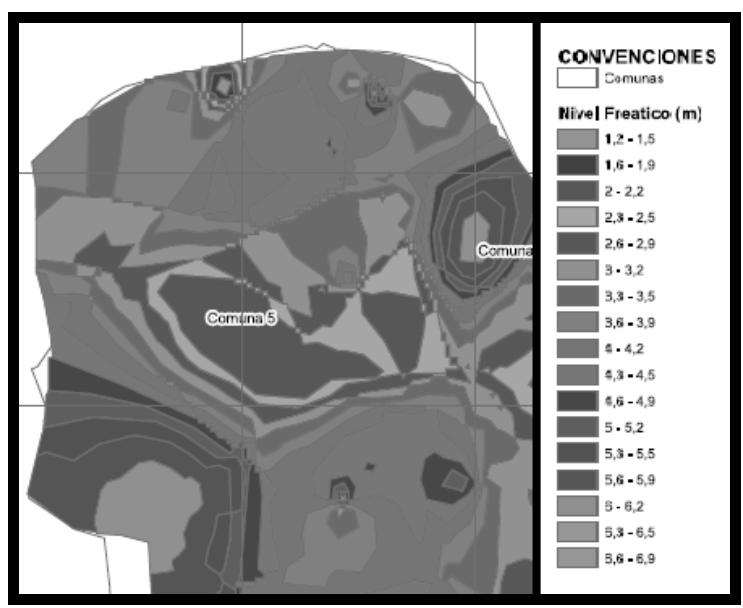

Figura 3. Nivel freático: comuna 5

Porcentaje de la humedad natural en el suelo de la ciudad de Valledupar.

A nivel general se encontró que la humedad natural del terreno, va de 6,3-11,8\%. En la comuna 2, se puede observar que el rango de 0,9 a $6,2 \%$ predomina. Siendo este el rango menor hallado.

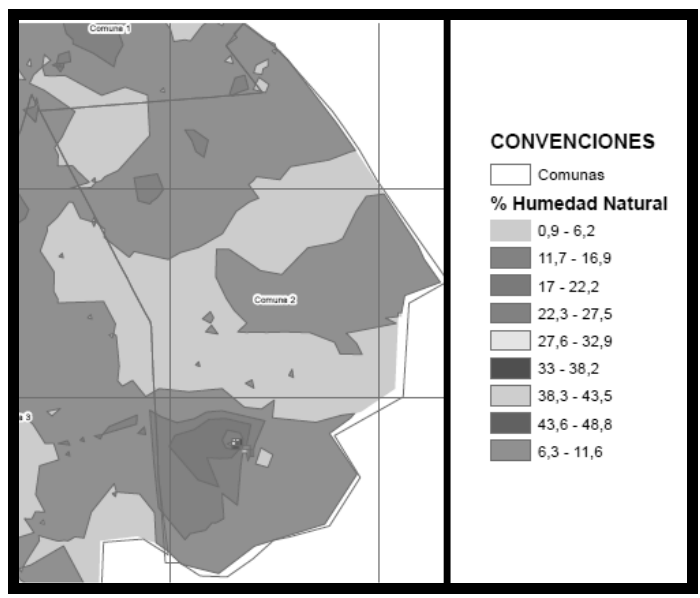

Figura 4. Humedad natural: comuna 2

Índice de plasticidad.

La comuna cuatro es la que presenta mayor variabilidad, mostrando rangos desde 0-2,5 a 10,2 - 12,6. Lo que indica que esta comuna tiene una mayor compresibilidad respecto a las otras.

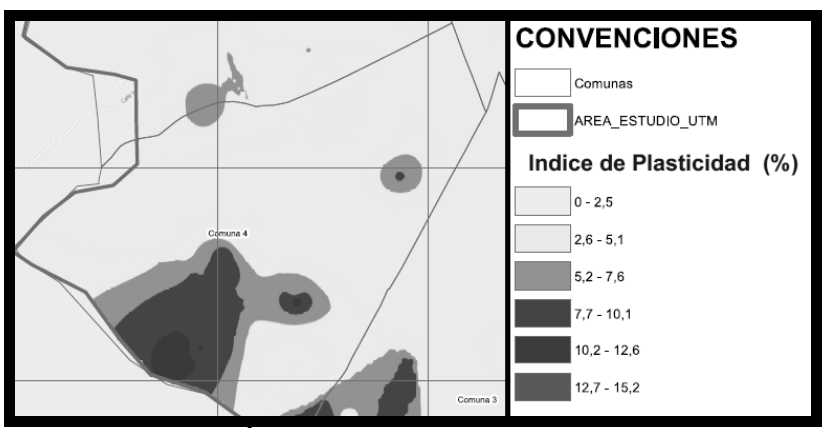

Figura 5. Índice de plasticidad: comuna 4 


\section{Capacidad de carga}

Para el análisis de este ítem se tomó en consideración la profundidad y los rangos con mayor y menor presencia según el área abarcada por cada uno [8].

\begin{tabular}{|c|c|c|}
\hline Profundidad & $\begin{array}{c}\text { Rangos con } \\
\text { mayor } \\
\text { presencia }\end{array}$ & $\begin{array}{c}\text { Rangos con } \\
\text { menor } \\
\text { presencia }\end{array}$ \\
\hline $50 \mathrm{~cm}$ & $8,1-9,1$ & $13,8-14,9$ \\
\hline $100 \mathrm{~cm}$ & $10,2-12,1$ & $22,1-23,9$ \\
\hline $150 \mathrm{~cm}$ & $\begin{array}{c}14,2-16,3, \\
16,4-18,5\end{array}$ & $25,3-27,4$ \\
\hline $200 \mathrm{~cm}$ & $20,3-22,9$ & $33,7-36,2$ \\
\hline $250 \mathrm{~cm}$ & $22,3-26$ & $45,1-48$ \\
\hline $300 \mathrm{~cm}$ & $29,6-32,2$ & $51,1-56,4$ \\
\hline $350 \mathrm{~cm}$ & $27,1-32,2$ & $74,9-95,4$ \\
\hline $400 \mathrm{~cm}$ & $35,5-36,9$ & $72-114,8$ \\
\hline
\end{tabular}

Tabla 3 capacidad de carga

\section{Ángulo de fricción interna.}

En forma general se puede observar en un rango de $31-32^{\circ}$, sin embargo, En la parte central de la comuna 4 (La victoria, Populandia, Villa Miriam Francisco de Paula) presenta variaciones desde 32 a $38^{\circ}$ y en la zona sur de la comuna 4 (Ciudadela 450 años, San Gerónimo y Girasoles) de 29-30

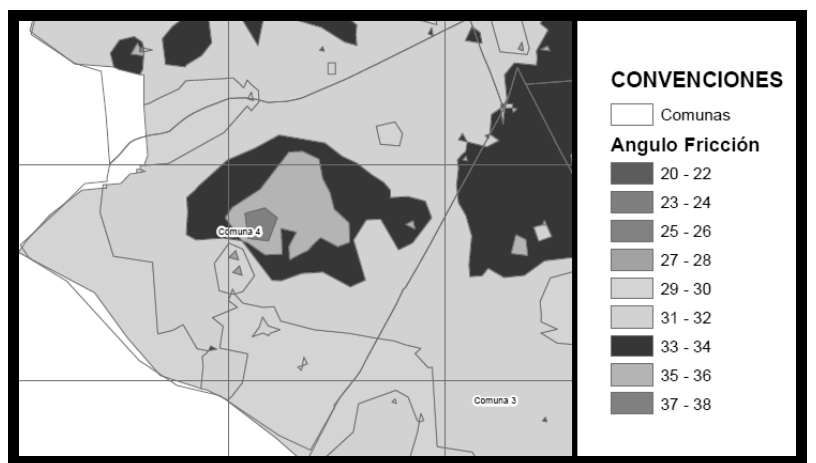

Figura 6. Ángulo de fricción interna: comuna 4 


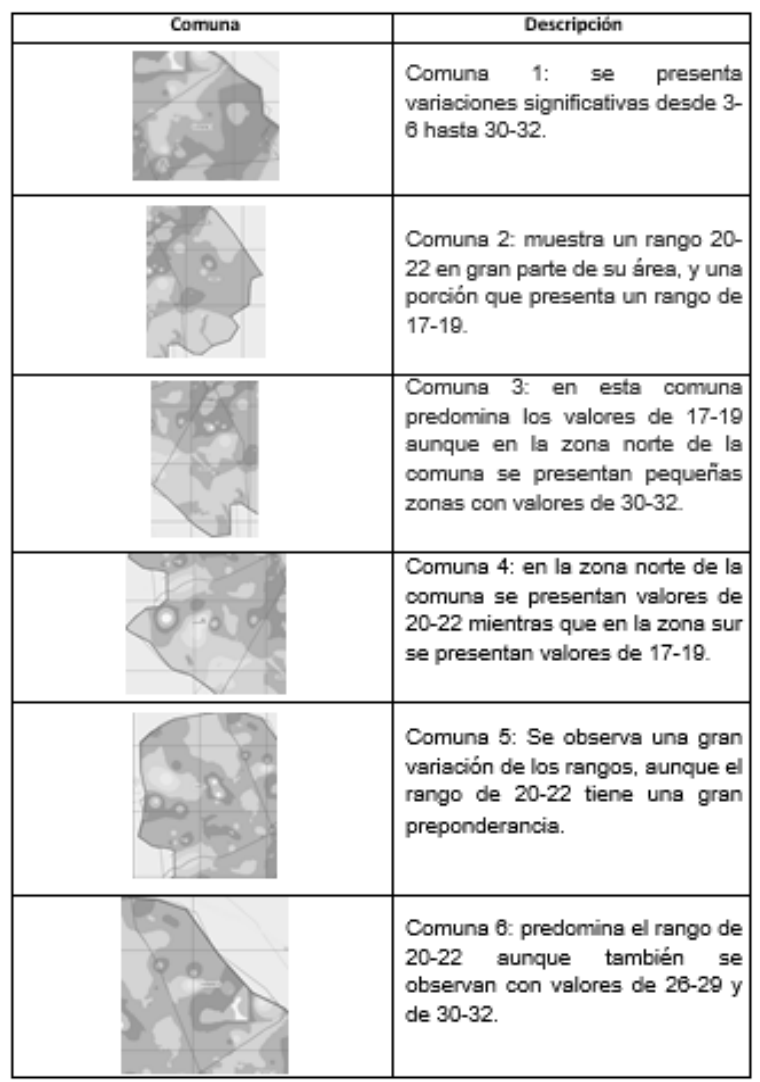

Tabla 4 Ensayo de penetración estándar (SPT)

Con el ensayo de penetración estándar se utiliza para hacer un reconocimiento geotécnico de los suelos, ya que permite caracterizar los suelos granulares (arenas y gravas arenosas), así como también en terrenos arcillosos conocer la humedad natural del terreno [11].

\section{Modelo de Winkler}

Se puede observar en el mapa que predominan los rangos de 1.061- 1.233 y $1.234-1.406$ mientras que en menos medida se encuentra el rango de 1.924- 2.096.

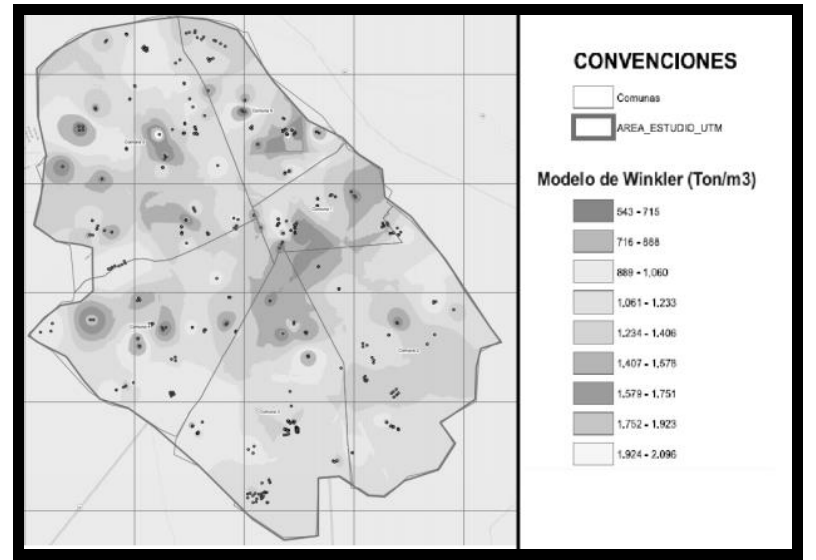

Figura 7. Modelo de Winkler 


\section{Velocidad de onda de corte}

La velocidad de onda de corte está relacionada con la rigidez y la densidad del terreno por donde es propagada, por lo que es un parámetro geofísico empleado para conocer la respuesta sísmica de los terrenos estudiados [11].

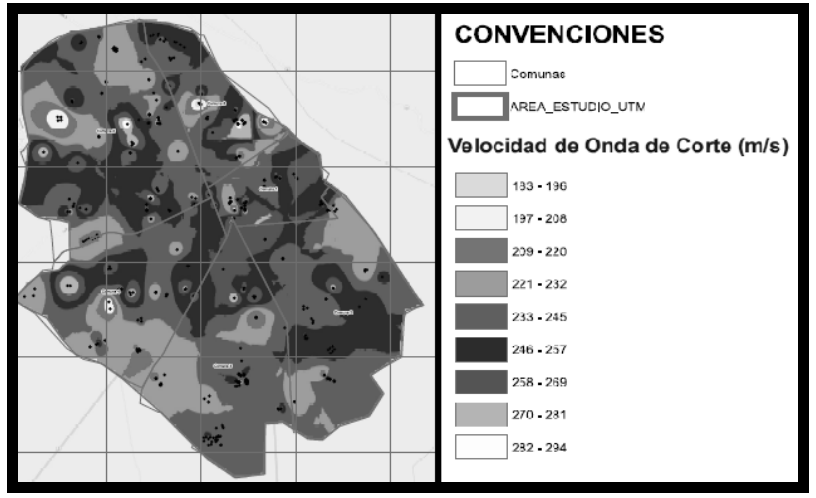

Figura 8. Velocidad de onda de corte

En todas las comunas se evidencio que existe una velocidad de onda de corte común que es de 233- 245 $\mathrm{m} / \mathrm{s}$.

\section{DISCUSIÓN (O ANÁLISIS DE RESULTADOS)}

Con la zonificación arrojada se pudo observar que en la ciudad priman los suelos arenosos y gravas, en cuanto a la capacidad de carga se tiene que a partir de los $0,5 \mathrm{~m}$ de profundidad hasta los 4 metros (profundidades estudiadas) se nota el aumento paulatino debido al aumento de profundidad, se define que la ciudad puede soportar cimientos para edificación de más de 10 niveles siendo la capacidades de carga a los $4 \mathrm{~m}$ superior a los 50 ton $/ \mathrm{m}^{2}$, en zonas con altos porcentajes de grava, estas áreas serán las de mayor incidencia a la hora de aumentar la cobertura de redes de acueducto y alcantarillado por lo que los presentes estudios ayudaran a observar con mayor claridad las condiciones de las áreas a tratar.

La mayor parte del área de estudio esta urbanizada y en las que no, están proyectadas para futuras construcciones por lo que el presente trabajo puede ser de ayuda como estudio previo.

Con la inclusión de los estudios en la realización del Sistema de Información Geográfico, se pudo comprobar que la ciudad es rica en aguas subterráneas al poder encontrar un nivel freático bajo a 1,2 $\mathrm{m}$ de profundidad, también cabe destacar el hecho de que se pudo encontrar el nivel freático en la mayoría de los de los estudios que se usaron para la base de datos usada en la zonificación.

\section{CONCLUSIONES}

En el área estudiada de la ciudad de Valledupar se encontraron en mayor porcentaje suelos de tipo SC, GW, ML, GP-GM, SP-SM, SM y SC-SM, estos suelos por sus características son usados para la construcción, por contener porcentajes altos de la combinación de gravas, arenas y arcillas, tener capacidades de carga altas y comportamiento mecánico buenos a excelentes.

Considerando la profundidad del nivel freático en la ciudad, hay zona en la que se encontró a niveles menores de $2 \mathrm{~m}$ de profundidad, en estas zonas es mejor evitar edificaciones de niveles altos, al igual que construcciones de estructuras de acueductos y alcantarillados como pozos de inspección, tanques elevados 
y alcantarillados pluviales, esto se presenta con mayor incidencia en pequeñas zonas de la comuna 1 y 5 que son cercanas al rio Guatapurí.

Esta zonificación geotécnica se hace como referente de estudios previos (preliminares) para obras de infraestructura como lo son las redes acueductos y alcantarillados, ya que los mapas cumplen a cabalidad con lo considerado como requisito previo de estudios de suelos en el RAS para obras de agua potable y saneamiento básico, en el que se requieren datos como la determinación de la clasificación de suelos, permeabilidad, nivel freático, entre otros estudios geotécnicos.

La investigación también tiene utilidad en otras áreas del conocimiento como son la ingeniería civil, la arquitectura y la ingeniería sanitaria; ya que nos permite conocer las condiciones ambientales del entorno en el que se desea trabajar antes de realizar una intervención.

Se deja como base la información del presente proyecto para la realización de proyecto similares o para la actualización de los mapas ya realizados. Se recomienda la creación de una aplicación como herramienta que permita a los estudiantes la inclusión de nueva información a la base de datos, tomadas en campo con la que se pueda ampliar y unificar la información sobre los suelos de la ciudad y que sea de fácil acceso para los futuros investigadores y/o comunidad universitaria.

\section{AGRADECIMIENTOS}

Los autores expresan su agradecimiento: A los laboratorios, V \& O Ingeniería S.A.S, Leixer Alfonso Rivero Londoño Estructuras Vías Geotecnia Concretos, Ernesto Altahona S.A.S, Armando Rivero Ingenieros S.A.S., por sus aportes para la realización de esta investigación.

\section{REFERENCIAS BIBLIOGRÁFICAS}

[1] Departamento Administrativo Nacional de Estadística. Resultados y proyecciones (2005-2020) disponible en:

http://www.dane.gov.co/files/investigaciones/poblacion/proyepobla06_20/ProyeccionMunicipios2005_2020.xl s

[2] Leas Cotés, C.A., Montoya López, A.J. Caracterización geotécnica del subsuelo en la zona norte de la cabecera municipal de Valledupar. Universidad popular del Cesar. Valledupar. (2017).

[3] ESRI. Introducción al SIG. Disponible en: http://resources.arcgis.com/es/help/gettingstarted/articles/026n0000000t000000.htm

[4] Bañon, L., \& Beviá, J. F. Manual de carreteras. Alicante: Ortiz e Hijos, Contratista de Obras, SA. (2000).

[5] López Flórez, L. V., \& Robayo González, F. A. (2007). Zonificación geotécnica preliminar del casco urbano del municipio de Barrancabermeja, Santander. Retrieved from https://ciencia.lasalle.edu.co/ing civil/205

[6] Guacaneme Berbeo, John Jairo (2006) "Zonificación de suelos en superficie de la ciudad de Tunja, Colombia," Épsilon: Iss. 6, Article 4.

[7] Braja, M. (2001). Fundamentos de Ingeniería Geotécnica. México: Thomson Learning.

[8] Alfaro, A. (2007, junio). Correlación entre el Valor N del Ensayo de Penetración Estándar y Velocidad de Ondas de Corte para Arcillas en Bogotá - Colombia. Revista Épsilon, (No. 8), 13-23

[9] Alarcón G., \& Jorge E. (2007). Integración de información geotécnica del Valle de Aburrá a un sistema de información geográfica SIG. Universidad Nacional, sede Medellín, Colombia. 
[10] Albarracín, Gómez, Alarcón, Sandoval.2009, "Zonificación Geotécnica de La Zona Centro del Área Urbana del Municipio de Sangamoso por medio de un SIG - Bogotá - Colombia".

[11] Guzmán, Martínez, Rubiano, Carlos Alvarado, Carrillo; Ministerio de Minas y Energía. 2001 "Zonificación Sismogeotécnica Indicativa del Área Metropolitana de Bucaramanga - Colombia ".

[12] Galli, C. Geología Urbana y suelo de fundación de Concepción y Talcahuano. Chile. Departamento de Geología y Mineralogía. Instituto Central de Química. U de C. 1967. 